\title{
Systematic Surveys in Informal Settlements: Challenges in Moving Toward Health Equity
}

\author{
David Vlahov
}

Published online: 29 October 2019

(C) The New York Academy of Medicine 2019

Over one billion people in the world live in informal settlements called slums. Slum populations continue to grow with the push-pull forces toward cities. Push toward cities can be due to war and adverse weather such as droughts. Pull is related to the opportunity to generate wealth through either the formal or informal economy. Without city resources and legal stature, governments have responded over time by having many of these settlements not included in overall urban plans that could be developed to provide trunk services, health care, education, and job training. The municipal and national policies that do not lift living conditions result in making unsafe living conditions and disparities in health. Views and actions are changing for a number of urban informal settlements, and such changes have been due to a number of factors. One of these factors is having credible data to document conditions.

Residents of informal settlements with nongovernmental agencies have moved toward essential early steps in generating a census of individual slums, and some census include assignment of street addresses. These data are critical for residents to be recognized and who may eventually qualify for voting and services. These are key steps toward advocacy for legal recognition and movement toward improving living conditions.

In addition to generating census data, efforts have been underway to document population health and conditions that affect health. Systematic surveys have been

D. Vlahov $(\bowtie)$

Yale School of Nursing, Orange, CT, USA

e-mail: David.Vlahov@yale.edu undertaken in a number of informal settlements. Some in positions of privilege and power possess a conception that these informal settlements are disorganized and unsafe with shifting boundaries and transient residents. Data can and have been moving these positions. An essential part of moving positions is to have credible data about residents, their needs, and their potential that can be addressed with opportunity and investment.

In this issue of the Journal, McNairy and colleagues [1] have reported on the high burden of noncommunicable diseases among a young slum population in Haiti. The data are compelling in part because they used a rigorous design. They used a multistage cluster area random sampling process, and they achieved impressive rates of participation. The sampling design was innovative in that they divided each of four informal settlements into blocks that had an estimated population count based on data from a prior GHESKIO survey and online high-resolution images. They created 111 geospatial waypoints, with the number of waypoints per block proportional to its estimated population. ArcGIS was used to randomly generate the waypoints with corresponding global positioning system (GPS) software within each block; commercial buildings and empty spaces were excluded. For each waypoint, community health workers (CHWs) followed a protocol to identify the closest five households.

Conducting surveys in informal settlements has challenges beyond sampling strategies [2, 3], and attention needs to be drawn to those challenges if we are to get the evidence that is needed to advance equitable population health. 


\section{References}

1. Margaret L. McNairy ML, Tymejczyk O,Rivera V, Seo G, Dorélien A, Mireille Peck P, Petion J, Walsh K, Bolgrien A, Nash D, Jean Pape J, Fitzgerald DW. High burden of noncommunicable diseases among a young slum population in Haiti. J Urban Health 2019

2. Lilford R, Kyobutungi C, Ndugwa R, Sartori J, Watson SI, Sliuzas R, et al. Because space matters: conceptual framework to help distinguish slum from non-slum urban areas. $B M J$
Glob Health. 2019;4(2):e001267. https://doi.org/10.1136 /bmjgh-2018-001267. eCollection 2019

3. Vlahov D, Agarwal SR, Buckley RM, Caiaffa WT, Corvalan $\mathrm{CF}$, Ezeh AC, et al. Roundtable on Urban Living Environment Research (RULER). J Urban Health. 2011;88(5):793-857. https://doi.org/10.1007/s11524-0119613-2.

Publisher's Note Springer Nature remains neutral with regard to jurisdictional claims in published maps and institutional affiliations. 